\title{
Non-invasive monitoring of paclitaxel and lenvatinib efficacy against anaplastic thyroid cancer in orthotopic SCID mouse models using small-animal FDG-PET/CT
}

\author{
MARIKO AOYAMA $^{1}$, HIROMITSU TAKIZAWA ${ }^{1}$, TAMAKI OTANI ${ }^{2}$, SEIYA INOUE $^{1}$, NAOYA KAWAKITA ${ }^{1}$, \\ MITSUHIRO TSUBOI $^{1}$, YOSHIMI BANDO ${ }^{3}$, HISANORI UEHARA ${ }^{3}$, KAZUYA KONDO $^{4}$ and AKIRA TANGOKU ${ }^{1}$ \\ ${ }^{1}$ Department of Thoracic, Endocrine Surgery and Oncology, Institute of Health Biosciences, \\ The University of Tokushima; ${ }^{2}$ Radioisotope Research Center, Tokushima University Graduate School; \\ ${ }^{3}$ Department of Pathology, Tokushima University Hospital; ${ }^{4}$ Department of Oncological Medical Services, \\ Institute of Health Biosciences, The University of Tokushima Graduate School, Tokushima 770-8503, Japan
}

Received November 12, 2019; Accepted June 10, 2020

DOI: 10.3892/or.2020.7720

\begin{abstract}
Anaplastic thyroid carcinoma (ATC) is a rare type of thyroid carcinoma with a poor prognosis. Thus, suitable preclinical tumor models are required for the development of new ATC therapies. In the present study, orthotopic tumor xenograft models were established using ATC cell lines and SCID mice, and tumor invasion and the effects of anticancer drugs were evaluated using positron emission tomography/computed tomography (PET/CT) to repeatedly and non-invasively monitor these models. Three ATC cell lines (8305c, 8505c, and ACT-1) were used. Their sensitivities to two anticancer drugs (paclitaxel and lenvatinib) were investigated. The $8505 \mathrm{c}$ cell line was orthotopically implanted into SCID mice, which were then divided into three groups: No chemotherapy, paclitaxel $(5 \mathrm{mg} / \mathrm{kg}$, administered intraperitoneally, every week), and lenvatinib ( $5 \mathrm{mg} / \mathrm{kg}$, oral route, every day) groups. PET/CT was performed and tumor growth and the effects of anticancer drugs based on tumor volume and fludeoxyglucose (FDG) uptake were evaluated. $8505 \mathrm{c}$ cells exhibited the highest sensitivity to the anticancer drugs. In mice implanted with $8505 \mathrm{c}$ cells, continuous increases in FDG uptake associated with tumor growth were detected on PET/CT in the group that received no chemotherapy. The tumor volume and FDG uptake increased by 91.5 - and 2.4-fold, respectively, within 2 weeks. The increase observed in tumor volume was 26.9- and 12.2-fold in the paclitaxel and lenvatinib groups, respectively, within 2 weeks. Furthermore, the increase in FDG uptake was 1.8 and 1.6-fold in the
\end{abstract}

Correspondence to: Dr Hiromitsu Takizawa, Department of Thoracic, Endocrine Surgery and Oncology, Institute of Health Biosciences, The University of Tokushima, 3-18-15 Kuramoto-cho, Tokushima 770-8503, Japan

E-mail: takizawa@tokushima-u.ac.jp

Key words: anaplastic thyroid carcinoma, orthotopic model, 18F-FDG PET/CT, paclitaxel, lenvatinib paclitaxel and lenvatinib groups, respectively, within 2 weeks. In our orthotopic SCID mouse model, tumor growth and the effects of anticancer drugs were repeatedly and non-invasively monitored using PET/CT. The present method is useful for the development of new ATC treatments.

\section{Introduction}

Anaplastic thyroid carcinoma (ATC) is one of the most aggressive human malignancies and has a poor prognosis (1-3). The average survival time of patients with ATC is approximately 3-4.5 months (1-3), with a 1-year survival rate of only $16-20 \%$ (1-3). Once diagnosed, a large number of ATC tumors cannot be radically resected because the disease is too advanced. There are currently no effective chemotherapy regimens for ATC, and the majority of patients diagnosed with ATC will succumb to the disease (1-3).

Doxorubicin has been used to treat ATC, but has only achieved modest effects (4,5). A few effective chemotherapy regimens and drugs have recently been approved as treatments for ATC. A phase 2 trial on paclitaxel for ATC patients reported an overall response rate (ORR) of 56\% (6). Another study demonstrated that the weekly administration of paclitaxel for ATC was effective and safe; the ORR was $21 \%$ and the clinical benefit rate was $73 \%$ (7). Lenvatinib is a multikinase inhibitor, and its targets are vascular endothelial growth factor receptors 1-3, fibroblast growth factor receptors 1-4, plated-derived growth factor receptor- $\alpha$, and the RET and KIT proto-oncogenes (8). In Japan, lenvatinib was approved as a treatment for radically unresectable thyroid cancer, including ATC, in 2015 (3). A phase 2, single-arm, open-label study on lenvatinib conducted in Japan reported that it resulted in a median progression-free survival period of 7.4 months, a median overall survival period of 10.6 months, and an ORR of $24 \%(3,8)$.

ATC is rare; i.e., it only accounts for $1-2 \%$ of all thyroid carcinomas $(9,10)$. The aggressive characteristics and rarity of ATC make it difficult to accumulate a sufficient number of cases for clinical studies aimed at developing new ATC treatments. The phase 2 trials on paclitaxel (6) and Lenvatinib (8) for ATC 
patients aforementioned included limited numbers of patients $(n=17-19)$. Therefore, the development and establishment of suitable preclinical tumor models that may be used to examine the effects of anticancer drugs against ATC are required.

Several animal models of thyroid carcinoma have been created, for example, models involving the subcutaneous or orthotopic implantation of cancer cells into immunodeficient mice (11-13). In 1889, Paget proposed the 'seed and soil' theory, in which an organ-specific site provides tumor cells with an appropriate environment for local growth and metastasis (14). Orthotopic tumor models may provide a more relevant biological setting than subcutaneous xenograft models in terms of the tumor microenvironment (12-18).

However, the main disadvantage of orthotopic tumor models is that difficulties are associated with assessing changes in tumor size, except at necropsy. Therefore, it is impossible to repeatedly and continuously monitor the same model. It was theorized that the use of small-animal positron-emission tomography/computed tomography (PET/CT) may overcome these disadvantages. Our group previously performed the non-invasive monitoring of the anticancer effects of cisplatin on lung cancer in an orthotopic SCID mouse model using ${ }^{18} \mathrm{~F}$-fludeoxyglucose (FDG) PET/CT (19). We also monitored the anticancer effects of cisplatin and erlotinib in an orthotopic SCID mouse model of lung cancer using both CT and PET/CT (20).

ATC typically exhibits high glucose metabolism and high FDG uptake in both primary and metastatic lesions $(21,22)$. The American Thyroid Association recommends the use of PET/CT to evaluate distant metastatic disease (21). FDG PET/CT may be used for initial staging, early evaluations of treatment responses, and follow-ups (22). In patients with ATC, PET/CT is useful for directing clinical management and evaluating the efficacy of therapy $(21,22)$.

In the present study, orthotopic tumor xenograft models were established using ATC cell lines and SCID mice. Tumor growth was also evaluated using PET/CT to repeatedly and non-invasively monitor these models. Furthermore, the antitumor effects of paclitaxel and lenvatinib against ATC were investigated with PET/CT.

\section{Materials and methods}

Cell lines. Three ATC cell lines were used: ACT-1, 8305c, and 8505c. The ACT-1 cell line was established at our institute (Tokushima University, Tokushima, Japan). The 8305c and $8505 \mathrm{c}$ cell lines were provided by the BioResource Center of RIKEN. The ACT-1 and 8305c cell lines were cultured in Roswell Park Memorial Institute-1640 medium (Nissui Pharmaceutical Co., Ltd.), and the 8505c cell line in Minimum Essential Medium (Sigma-Aldrich; Merck KGaA) supplemented with $10 \%$ fetal bovine serum (Bio-West), $100 \mathrm{IU} / \mathrm{ml}$ of penicillin, and $100 \mu \mathrm{g} / \mathrm{ml}$ of streptomycin (Gibco; Life Technologies; Thermo Fisher Scientific, Inc.). Cells were maintained at $37^{\circ} \mathrm{C}$ in a humidified incubator equilibrated with $5 \% \mathrm{CO}_{2}$ and $95 \%$ air. Cells were grown in tissue culture flasks, and passaged at 5- to 7-day intervals at $80 \%$ confluence. Cell morphology and viability were monitored by microscopic observations. All cell lines were used after being propagated between 10 and 20 times. Information on all cell lines was confirmed and checked using the International Cell Line
Authentication Committee (https://iclac.org/) and ExPASy Cellosaurus databases (https://web.expasy.org/cellosaurus/).

Drugs. Paclitaxel was purchased from Fujifilm Wako Pure Chemical Corporation and lenvatinib from LC Laboratories.

Cell viability after drug exposure. Cells (ACT-1 cells: 4x1033 $8305 \mathrm{c}$ and $8505 \mathrm{c}$ cells: $2 \times 10^{3}$ ) were seeded on 96 -well plastic culture plates $(100 \mu \mathrm{l} /$ well). ACT-1 cells were maintained for $48 \mathrm{~h}$, and $8305 \mathrm{c}$ and $8505 \mathrm{c}$ cells for $24 \mathrm{~h}$ at $37^{\circ} \mathrm{C}$ in a humidified incubator with $5 \% \mathrm{CO}_{2}$ and $95 \%$ air. They were treated with the intended doses of each agent for $48 \mathrm{~h}$. After the incubation period, 3-(4,5-dimrthylthiazolyl-2)-2,5-diphenyltetrazolium bromide (MTT; Nacalai Tesque, Inc.) was added at a final concentration of $0.5 \mathrm{mg} / \mathrm{ml}$, and cells were incubated for $4 \mathrm{~h}$ under the same conditions as aforementioned. Dimethyl sulfoxide (Nacalai Tesque, Inc.) was added, and spectrophotometric absorbance was measured at $535 \mathrm{~nm}$ with a microplate reader (Spectra Max i3; Molecular Devices, LLC) and calculated using the supplied software (SoftMax Pro, version 7). The control sample was untreated and incubated in an equal volume of medium (100 $\mu \mathrm{l} /$ well). Each experiment was performed three times independently, and the mean values for the three independent experiments were calculated. The concentrations of the agents at which cell growth was inhibited by $50 \%$ ( $\mathrm{IC}_{50}$ values) were assessed using growth-inhibition curves.

Animals. As described in our previous studies involving an orthotopic mouse model of lung cancer $(19,20)$, female nine SCID mice (CB-17/Icr-scidJcl; CLEA Japan, Inc.) (age, 6-8 weeks; mean body weight, $20 \mathrm{~g}$ ) were purchased and maintained at our institution's laboratory for animal experiments (Tokushima University, Tokushima, Japan). Animals were housed in Micro-Isolator cages on a layer of wood shavings at $22^{\circ} \mathrm{C}$ and $55 \%$ humidity under a fixed 12-h light/dark regime. A basic diet (MF; Oriental Yeast Co.) and water were available ad libitum. All studies were performed in accordance with the guidelines established by the Tokushima University Committee on Animal Care and Use. At the end of each in vivo experiment, mice were anesthetized and then euthanized humanely by vertebral dislocation (20) or, mice were euthanized when they had lost $>20 \%$ of their baseline body weight or had signs of tracheal compressive symptoms. Death was confirmed by observation of respiratory and cardiac arrest. All experimental protocols were reviewed and approved by the Animal Research Committee of the University of Tokushima, Japan. All sections of this study adhered to the ARRIVE Guidelines for reporting animal research (23).

Orthotopic mouse model of anaplastic thyroid cancer. On the day of tumor implantation, cells were trypsinized, centrifuged (140 x g, $5 \mathrm{~min})$, and resuspended in serum-free media to produce ACT-1, 8305c, and 8505c cell suspensions with a cell density of $5 \times 10^{5} / \mu \mathrm{l}$ cells, and $0.4 \mathrm{mg} / \mu \mathrm{l}$ Matrigel (Collaborative Biochemical Products) was then added to each cell suspension. Cell suspensions were maintained on ice until the implantation procedure. As described in our previous studies involving an orthotopic mouse model of lung cancer $(19,20), 9$ mice were fully anesthetized via the inhalation of $1.5 \%$ isoflurane, before being injected with tumor cells. A 1-cm transverse incision was produced along the middle of the throat. The salivary 
glands were reflected laterally, the trachea was exposed, and the overlaying strap muscles were dissected away from the right thyroid lobe. Once the right thyroid lobe was exposed, the cell suspension $\left(5 \times 10^{5}\right.$ cells in $2 \mu \mathrm{l}$ of medium (Minimum Essential Medium; Sigma-Aldrich; Merck KGaA) was injected into the right thyroid gland using a Hamilton syringe (Thermo Fisher Scientific, Inc.) attached to a 30-gauge needle. After the injection of the cell suspension, the salivary glands were repositioned and the skin incision was closed with 3-0 silk sutures. After the implantation, all mice were weighed and monitored for signs of distress regularly every week.

FDG PET/CT-based analysis of tumor volumes and maximum standardized uptake value $\left(S U V_{\max }\right)$. As described in our previous studies $(19,20)$, FDG PET/CT scans were performed with a Siemens Inveon small-animal PET scanner (Siemens Healthcare). Mice that had been orthotopically implanted with $8505 \mathrm{c}$ cells were fasted for $12 \mathrm{~h}$, but were allowed free access to water. Their body weights were measured, and mice were then anesthetized via the inhalation of 1.5-2.0\% isoflurane, before $10 \mathrm{MBq} / 0.1-0.2 \mathrm{ml}$ FDG was injected via a tail-vein catheter. The whole body of each mouse was scanned using CT [field of view: $32.0 \times 32.0 \times 48.1 \mathrm{~mm}^{3}$ ]. PET data were acquired for $20 \mathrm{~min}$ after a delay of $40 \mathrm{~min}$ to allow for FDG uptake. PET/CT images were analyzed with PMOD version 3.7 (PMOD Technologies LLC). In each PET/CT scan, the metabolic tumor volume (MTV) was calculated based on the volume of interest (VOI), which was drawn manually around the primary tumor on PET/CT images after referring to CT images. Since the boundary between the thyroid gland and surrounding structures was unclear on CT alone, MTV was used as the tumor volume in the present study. On fused PET images, $\mathrm{SUV}_{\max }$ was calculated from the maximum voxel value $(\mathrm{Bq} / \mathrm{ml})$ in the VOI. Field-of-view of coronal-view in the PET-CT images was $10 \times 10 \mathrm{~cm}$, and the scale was calculated based on it.

Evaluation of tumor growth in mice that had been orthotopically implanted with 8505 c cells. The period of the tumor growth evaluation study was set from the day of tumor implantation to 5 weeks after implantation. In weeks 3 to 5 after the implantation of $8505 \mathrm{c}$ cells, PET/CT measurements on the no treatment group $[n=3]$ were performed every 7 days.

Evaluation of the inhibition of tumor growth in mice that had been orthotopically implanted with 8505 c cells. The period of the tumor growth inhibition study was set from the day of tumor implantation to 4 weeks after implantation, since the body weight loss of the non-treated mice was 18 and $28 \%$ at 4 and 5 weeks after the implantation procedure, respectively.

Two weeks after the implantation of $8505 \mathrm{c}$ cells, 6 mice were subjected to PET/CT measurements. To select drug doses, we referred to a study by Jing et al (24). The interval of paclitaxel administration was modified according to our preliminary examination. Jing et al administered paclitaxel twice per week. However, a sufficient antitumor effect was confirmed even once a week and marked weight loss was observed when paclitaxel was administered twice per week in the present study. Therefore, we adopted a weekly administration regimen for the paclitaxel group $[\mathrm{n}=3,5 \mathrm{mg} / \mathrm{kg}$, intraperitoneal administration, every week], and daily administration regimen for the lenvatinib group $[\mathrm{n}=3,5 \mathrm{mg} / \mathrm{kg}$, oral administration, every day]. PET/CT measurements were performed every 7 days from 2 to 4 weeks after the implantation procedure.

Histological examinations. We sacrificed the mice and en bloc resected the bilateral thyroid glands, bilateral salivary glands, trachea, bilateral lungs, and mediastinal region. The tumors implanted in the thyroid and surrounding organs were cut into 1-2-mm-thick pieces in the horizontal direction. These pieces were fixed with $10 \%$ formalin at $22^{\circ} \mathrm{C}$ for one week and embedded in paraffin blocks. Paraffin sections that had been stained with hematoxylin and eosin (H\&E) were examined under an Olympus BX51 polarizing microscope (Olympus Corporation; magnifications, $\mathrm{x} 20$ and $\mathrm{x} 200$ ). It was not possible to perform a pathological examination on the mice subjected to PET/CT because their removal from the PET/CT facility was prohibited in order to prevent radiation exposure.

Statistical analysis. Due to animal protection concerns, experiments were conducted with the minimum sample size. Statistical methods based on the standard maximum likelihood were not applicable. As an alternative, the significance of differences in tumor volume, $\mathrm{SUV}_{\max }$, and body weight were assessed using the mean data for three mice.

\section{Results}

Cell viability after drug exposure. The results of the cell viability assay are presented in Fig. 1. The paclitaxel and lenvatinib treatments both dose-dependently inhibited cell viability in all cell lines. Among the three cell lines assessed, 8505c cells exhibited the highest sensitivity and lowest $\mathrm{IC}_{50}(250 \mathrm{nM} / \mathrm{l})$ to paclitaxel (Fig. 1A). All cell lines displayed similar levels of sensitivity to lenvatinib (Fig. 1B).

Tumor growth and $S U V_{\max }$ in the 8505 c cell-based orthotopic mouse model. In the orthotopic $8505 \mathrm{c}$ cell-based model mice, there was no FDG uptake in thyroid tissue 3 weeks after the implantation procedure on PET/CT (Fig. 2A-a). FDG uptake was observed in the right thyroid lobe 4 weeks after the implantation procedure on PET/CT, which also revealed lateral deflection of the trachea due to increases in the size of the tumors 4 weeks after the implantation procedure (Fig. 2A-b). Mean tumor volumes were 44.6- and 91.5-fold higher at 4 and 5 weeks after the implantation procedure, respectively, than those at 3 weeks after the implantation procedure (Fig. 2B-a). The maximum tumor volume and diameter at 5 weeks after the implantation were $1042.9 \pm 58.3 \mathrm{~mm}^{3}$ and $13.3 \pm 0.6 \mathrm{~mm}$, respectively (Fig. 2A-c). FDG uptake increased by 2.8 -fold from 3 to 4 weeks after the implantation procedure; however, decreased FDG uptake was observed after 5 weeks (Fig. 2B-b). On coronal PET/CT images, FDG uptake inside the tumors had decreased at 5 weeks (Fig. 2A-c). The body weights of the mice decreased to a maximum $28 \%$ (Fig. 2B-c). Gross examinations of the tumors revealed that they were approximately $1.5 \mathrm{~cm}$ in diameter (Fig. 3A), and necrotic changes were pathologically observed in the central parts of the tumors (Fig. 3B-a) 5 weeks after the implantation procedure. The mice in the tumor growth evaluation experiment were anesthetized and then euthanized humanely by vertebral dislocation at the end of the experiment 5 weeks 

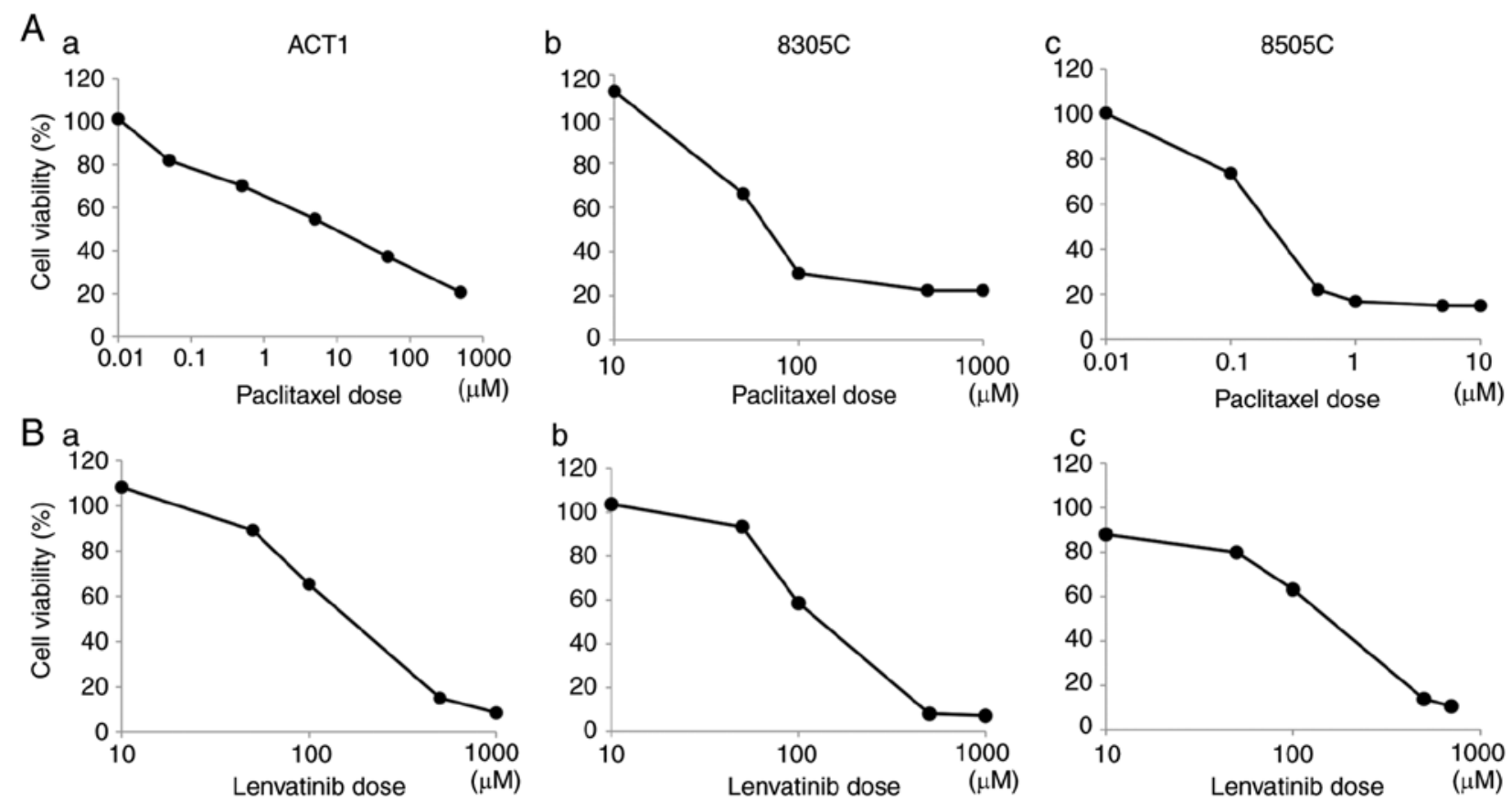

Figure 1. Effects of chemotherapy drugs (paclitaxel and lenvatinib) on cell viability according to the MTT assay. (a) ACT1, (b) 8305c, and (c) 8505c cells were treated with (A) paclitaxel or (B) lenvatinib.

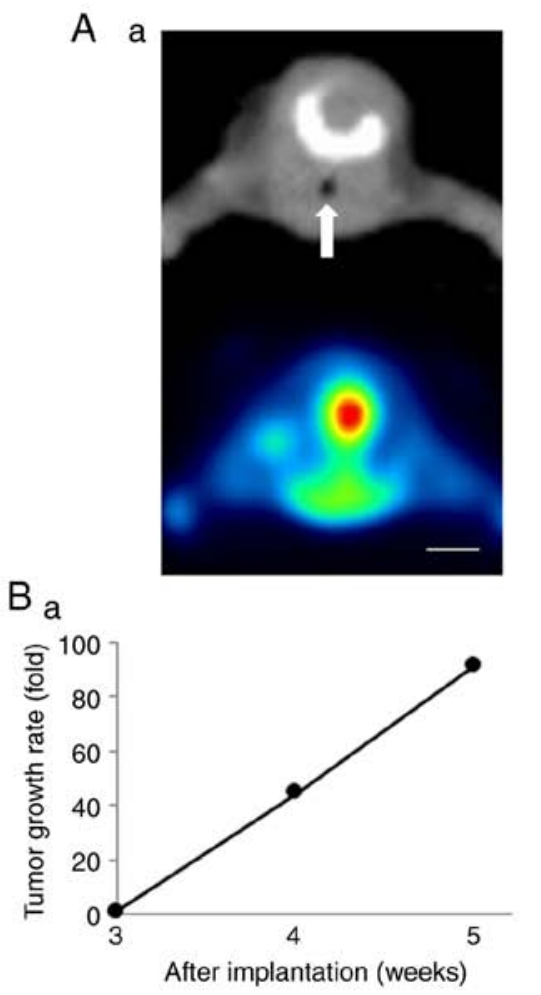

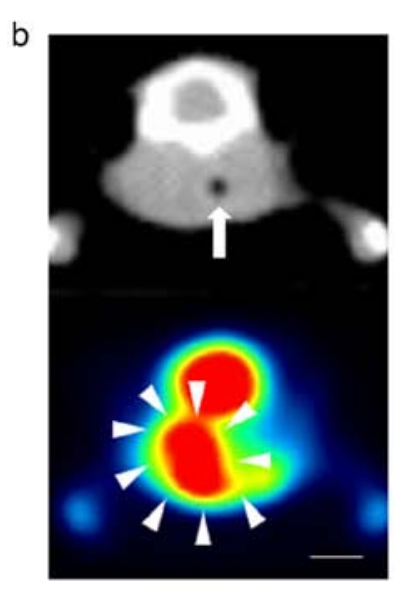

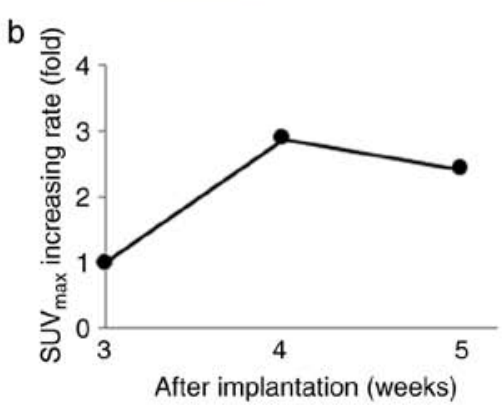

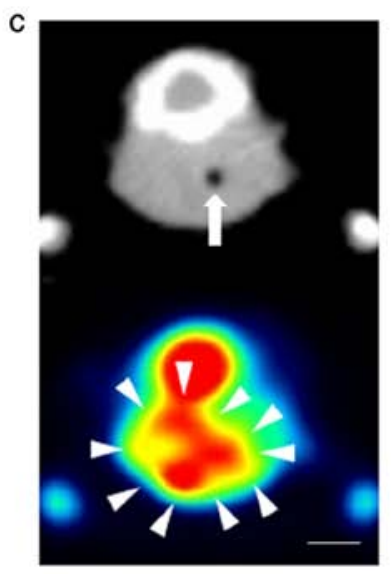

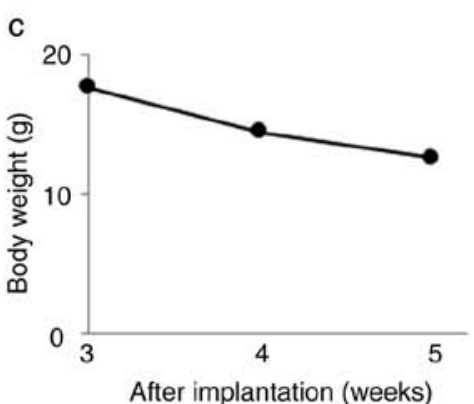

Figure 2. CT and PET/CT findings obtained in the no chemotherapy group. (A) Coronal-view CT and PET/CT images of tumors derived from $8505 \mathrm{c}$ cells in the right thyroid lobe. The arrows in the upper images indicate the trachea, and the arrow heads in the lower images indicate tumors. Scale bars, $5 \mathrm{~mm}$ (a) Three weeks after implantation, (b) 4 weeks after implantation, and (c) 5 weeks after implantation. (B) Quantitative PET/CT imaging data obtained from mice implanted with 8505 c cells. (a) Tumor growth rate from 3 to 5 weeks after implantation. Values on the vertical axis represent the tumor growth rate based on the tumor volume at 3 weeks after implantation. (b) The increasing rate of $\operatorname{SUV}_{\max }$ (FDG uptake) from 3 to 5 weeks after implantation. Values on the vertical axis represent the increasing rate of $\mathrm{SUV}_{\max }$ based on the $\mathrm{SUV}_{\max }$ value at 3 weeks after implantation. (c) Body weight from 3 to 5 weeks after implantation. CT, computed tomography; $\mathrm{PET} / \mathrm{CT}$, positron emission tomography/computed tomography; $\mathrm{SUV}_{\max }$, maximum standardized uptake value; FDG, fludeoxyglucose.

after the implantation procedure, and there was no accidental death during the experiment. Orthotopic implantation was also performed with ACT-1 and 8305c cells as well as $8505 \mathrm{c}$ cells. Although tumors were pathologically diagnosed in these mouse 
A

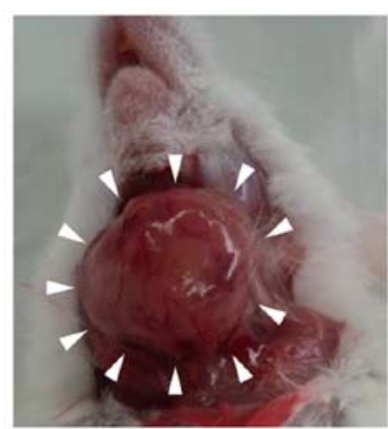

B

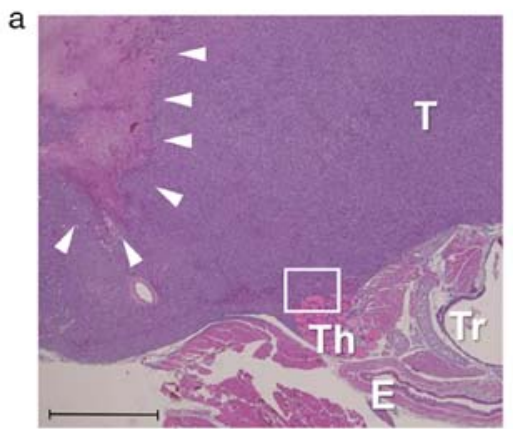

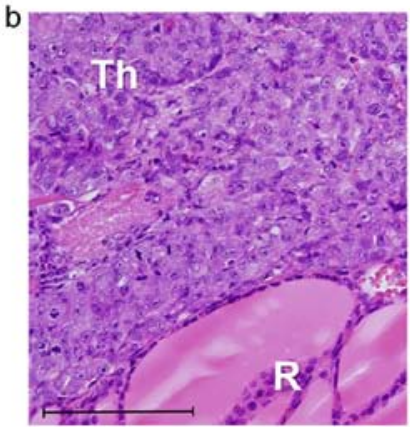

Figure 3. Histological findings. (A) Gross examination of a tumor derived from $8505 \mathrm{c}$ cells performed 5 weeks after implantation. (B-a) Histological sections of tumors derived from $8505 \mathrm{c}$ cells. H\&E staining (magnification, x20). Th, thyroid lobe; T, tumor; E, esophagus; Tr, trachea; arrows, necrotic changes in the central part of the tumor. scale bar, 1,000 $\mu \mathrm{m}$. (B-b) Magnified image of the white square presented in B-a. H\&E staining (magnification, x200). Th, thyroid lobe; T, tumor. Scale bar, $100 \mu \mathrm{m}$. H\&E, hematoxylin and eosin.

A a

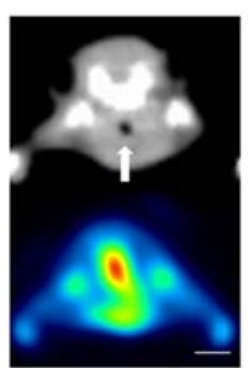

B

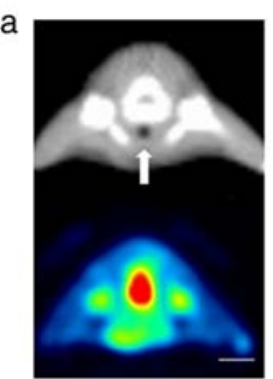

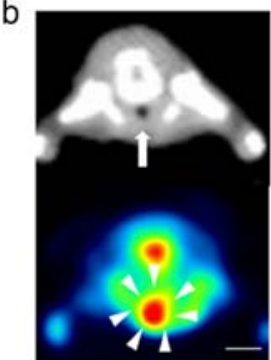

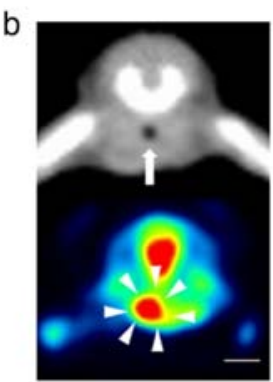

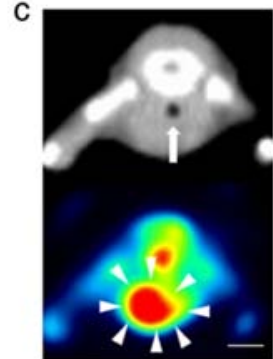

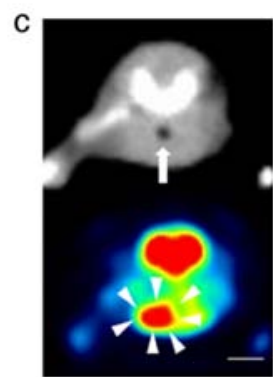

Figure 4. Coronal CT and PET/CT images of orthotopic mice in treated groups that had been implanted with 8505c cells. The arrows in the upper images indicate the trachea, and the arrow heads in the lower images indicate the tumors. Scale bar, $5 \mathrm{~mm}$. (A) Paclitaxel group and (B) lenvatinib group, (a) 2 weeks after implantation, (b) 3 weeks after implantation, and (c) 4 weeks after implantation. CT, computed tomography; PET/CT, positron emission tomography/computed tomography.
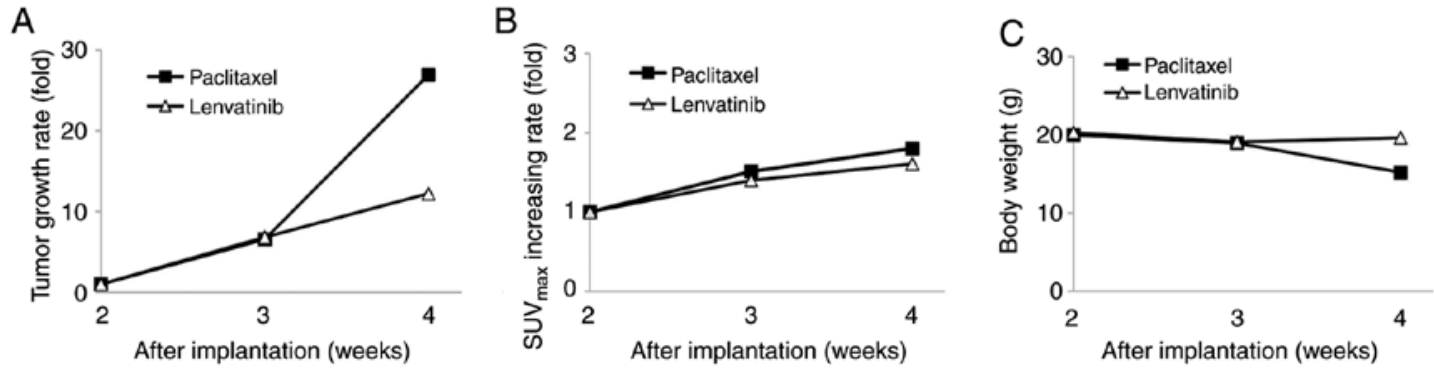

Figure 5. Quantitative PET/CT imaging data obtained from mice in treated groups that were implanted with 8505c cells. (A) Tumor growth rate from 2 to 4 weeks after implantation. Values on the vertical axis represent the tumor growth rate based on the tumor volume at 2 weeks after implantation. (B) The increasing rate of $\mathrm{SUV}_{\max }\left(\mathrm{FDG}\right.$ uptake) from 2 to 4 weeks after implantation. Values on the vertical axis represent the increasing rate of $\mathrm{SUV}_{\max }$ based on the SUV $\mathrm{V}_{\max }$ value at 2 weeks after implantation. (C) body weight from 2 to 4 weeks after implantation. PET/CT, positron emission tomography/computed tomography; $\mathrm{SUV}_{\text {max }}$, maximum standardized uptake value; FDG, fludeoxyglucose.

models, no clear macroscopic tumor formation was observed 6 weeks after the implantation of ACT-1 or 8305c cells (data not shown). Since tumors derived from ACT-1 or $8305 \mathrm{c}$ cells grew markedly slower than those derived from $8505 \mathrm{c}$ cells, the 
mouse models involving the implantation of ACT-1 or $8305 \mathrm{c}$ cells were not considered to be appropriate for assessing the antitumor effects of chemotherapy. Thus, PET/CT-based examinations on tumor growth and the antitumor effects of chemotherapy were only performed using the $8505 \mathrm{c}$ cell-based orthotopic mouse model.

Antitumor effects of paclitaxel and lenvatinib in the $8505 \mathrm{c}$ cell-based orthotopic mouse model according to FDG PET/CT imaging. We examined the antitumor effects of paclitaxel and lenvatinib in mice that had been implanted with $8505 \mathrm{c}$ cells using PET/CT. No tumors were detected on PET/CT 2 weeks after the implantation procedure in the paclitaxel or lenvatinib group (Fig. 4A-a and B-a). FDG uptake was observed at the putative tumor sites in both treated groups 3 weeks after the implantation procedure (Fig. 4A-b and B-b). The size of the tumors on coronal PET/CT images increased from 3 to 4 weeks after implantation in both treated groups, whereas tumor growth rates were markedly slower than that in the no treatment group (Fig. 4A-c and B-c). Throughout the treatment period, the increases observed in tumor volume and FDG uptake in the control group were suppressed in both treated groups. In the paclitaxel group, the mean tumor volumes were 6.6- and 26.9-fold higher at 3 and 4 weeks after the implantation procedure, respectively, than that at 2 weeks after the implantation procedure (Fig. 5A). In the paclitaxel group, the maximum tumor volume and diameter at 4 weeks after the implantation were $595.4 \pm 190.0 \mathrm{~mm}^{3}$ and $8.7 \pm 1.5 \mathrm{~mm}$, respectively (Fig. $4 \mathrm{~A}-\mathrm{c}$ ). In the lenvatinib group, the mean tumor volumes were 6.8- and 12.2-fold higher at 3 and 4 weeks after the implantation procedure, respectively, than that at 2 weeks after the implantation procedure (Fig. 5A). In the lenvatinib group, the maximum tumor volume and diameter at 4 weeks after the implantation were $216.2 \pm 83.9 \mathrm{~mm}^{3}$ and $6.7 \pm 0.6 \mathrm{~mm}$, respectively (Fig. 4B-c). In the paclitaxel group, FDG uptake increased by 1.5 - and 1.8-fold from 2 to 3 weeks and from 2 to 4 weeks after the implantation procedure, respectively. In the lenvatinib group, FDG uptake increased by 1.4- and 1.6-fold from 2 to 3 weeks and from 2 to 4 weeks after the implantation procedure, respectively (Fig. 5B). A marked antitumor effect was observed from 3 to 4 weeks after the implantation procedure in the lenvatinib group (Fig. 5A and B). The maximum body weight loss of the mice in the lenvatinib group was $3 \%$, whereas the maximum weight loss in the paclitaxel group was $24 \%$ at 4 weeks after the implantation procedure (Fig. 5C). The mice in the tumor growth inhibition experiment were euthanized at 4 weeks after the implantation procedure.

\section{Discussion}

In the present study, the growth of tumors in an orthotopic mouse model that had been implanted with $8505 \mathrm{c}$ cells was monitored using FDG PET/CT. Th mean tumor volumes were 44.6- and 91.5-fold higher 4 and 5 weeks after the implantation procedure, respectively, than that 3 weeks after the implantation procedure. The rapid growth of these tumors reflects the aggressive characteristics of ATC. Furthermore, the trachea, esophagus, and other surrounding tissues were displaced as the tumors grew, which is consistent with the clinical course of ATC (25). Conversely, although FDG uptake increased from 3 to 4 weeks after the implantation procedure, it decreased from 4 to 5 weeks. FDG uptake was also only observed at the margins of the tumors, and was not detected in the center of the tumors on PET/CT images obtained 5 weeks after the implantation procedure. Histological examinations on tumors performed 5 weeks after the implantation procedure revealed necrotic changes in the central parts, which were surrounded by viable tumor cells. Central tumor necrosis is one of the typical histological changes associated with ATC $(25,26)$. The decrease observed in tumor FDG uptake from 4 to 5 weeks after the implantation procedure was considered to have been caused by necrotic changes. Therefore, the tumor volume, rather than FDG uptake, needs to be measured as an index of tumor growth in orthotopic mouse models involving the implantation of $8505 \mathrm{c}$ cells.

We previously reported that FDG PET/CT is useful for monitoring antitumor effects in an orthotopic lung cancer model $(19,20)$. In these studies, the tumor volume was easy to calculate based on CT images since the CT values of the tumors and lung fields were different $(19,20)$. Conversely, in the present study, the boundaries between the tumors and the surrounding structures of the thyroid gland were unclear on CT images because their CT values were similar. Therefore, MTV, which was calculated based on the area of FDG uptake, was adopted as the tumor volume in the present study. MTV has been reported as a marker that increases the accuracy of prognostication and clinical staging in human tumors (27-29). Manohar et al reported that MTV reflected a higher tumor burden for patients receiving multikinase inhibitor therapy for metastatic radioiodine-refractory differentiated thyroid cancer (29). PET and CT were both considered to be important for evaluating tumors in orthotopic xenograft models involving the implantation of ATC cell lines into SCID mice.

The antitumor effects of paclitaxel and lenvatinib in an orthotopic mouse model that had been implanted with $8505 \mathrm{c}$ cells were examined using PET/CT. In the chemotherapy groups, FDG uptake and tumor volume were both lower than those in the no chemotherapy group. Therefore, the present study demonstrated that FDG uptake and MTV may be used to assess the effects of anticancer drugs in orthotopic mouse models involving the implantation of $8505 \mathrm{c}$ cells. A previous study reported that lenvatinib exerted antitumor and antiangiogenic effects in numerous thyroid cancer xenograft models, including ATC models (30). Furthermore, Jing et al demonstrated the effects of lenvatinib and paclitaxel in subcutaneous xenograft models of ATC (24). The effects of lenvatinib and paclitaxel in an orthotopic tumor xenograft model of ATC were confirmed in the present study. In addition, the effects of the two drugs were objectively demonstrated using PET/CT. This is the first study, to the best of our knowledge, to demonstrate the antitumor effects of paclitaxel and lenvatinib in an orthotopic tumor xenograft model of ATC with PET/CT.

Orthotopic tumor models provide a suitable preclinical environment for evaluating the effects of anticancer drugs (15). The main disadvantage of using orthotopic tumor models for this purpose is the difficulty associated with monitoring tumor size. Small-animal PET/CT was employed to overcome this issue. The use of PET/CT had the following advantages. The main advantage was that any changes in tumor size may be continuously evaluated in each mouse using PET/CT. By monitoring the changes in tumor size that occurred in each individual mouse, we were able to clearly assess tumor growth and the 
therapeutic effects of the anticancer drugs. Conversely, previous studies $(16,17)$ that evaluated the therapeutic effects of anticancer drugs using orthotopic thyroid cancer animal models required the sacrifice of more than 6 mice at each time-point to ensure the quality of the data obtained; however, the resultant data had no real continuity. Nehas et al revealed that anti-BRAFV600E therapy inhibited tumor growth in an orthotopic mouse model that had been implanted with $8505 \mathrm{c}$ cells (16). Forty-eight mice were orthotopically implanted with ATC cells and divided into 8 groups of 6 mice for the purpose of establishing the time course of tumor progression and the response to late therapeutic interventions with PLX4720, which selectively inhibits BRAFV600E. Each week, 6 mice were sacrificed, and tumor growth was evaluated to assess the effectiveness of PLX4720. Cha et al (17) investigated the effects of a perioperative treatment with echinomycin, which is a hypoxia-inducible factor-1 $\alpha$ inhibitor, using an orthotopic surgical mouse model of thyroid cancer. In the latter study, 60 mice were implanted with thyroid cancer cell lines. Mice were divided into four groups of 15 mice: A control group, surgical group, echinomycin group, and surgical and echinomycin group, and were sacrificed to evaluate the effects of each treatment. In the present study, only 9 mice were required to evaluate tumor growth and the effects of the two drugs. Therefore, our PET/CT-based method appears to be an ideal preclinical experimental system from the point of view of animal welfare.

We were also able to detect tumor formation earlier than previous studies involving mouse models in which $8505 \mathrm{c}$ cells were orthotopically implanted. Nucera et al demonstrated that tumors became palpable 35 days after the implantation of $8505 \mathrm{c}$ cells (12). However, by using PET/CT, FDG uptake on the right side of the trachea was observable 21 days after the implantation procedure in the treatment group. Thus, whether each orthotopic mouse model was suitable was confirmed at an early stage.

Moreover, continuous detailed examinations of tumor growth and its effects on the surrounding organs were possible in the same model using PET/CT. Previous studies demonstrated that orthotopic models involving the implantation of $8505 \mathrm{c}$ cells produced large tumors, which resulted in cachexia and death by 35 days after the implantation procedure $(12,16)$. Similar findings to those described in these studies were reproduced in our models, and we were able to observe gradual increases in tumor size that displaced the trachea and surrounding tissues using PET/CT. However, when the neck tumor exceeds a certain size, esophageal compression by the tumor causes insufficient oral intake and rapid weight loss. Therefore, conducting experiments within the range of weight loss allowed by the experimental protocols requires careful experimental period setting and mouse monitoring.

Paclitaxel and lenvatinib both improved the prognosis of ATC patients. However, the effects of these therapies are limited. The combination of chemotherapy and molecular targeted agents was recently evaluated in various cancer fields (31). Combination therapy with paclitaxel and lenvatinib may improve the treatment outcomes of ATC, and our model has the potential to become an effective tool for the development of this new therapy.

In conclusion, in the present study, the utility of FDG PET-CT for repeated and non-invasive evaluations of tumor progression and the effects of antitumor drugs (paclitaxel and lenvatinib) in orthotopic ATC models were demonstrated. The methods presented in this study may facilitate the development of new ATC treatments.

\section{Acknowledgments}

The authors would like to thank Mr. Toshio Yamaguchi and Ms Kana Tominaga for their technical assistance.

\section{Funding}

No funding was received.

\section{Availability of data and materials}

The datasets used and analyzed during the present study are available from the corresponding author on reasonable request.

\section{Authors' contributions}

HT had full access to all data in the study and takes responsibility for the integrity of the data and accuracy of the data analysis. MA, TO, and SI performed the experiments, and NK analyzed the data. MT, KK, and AT designed the study. YB and HU contributed to histological examinations. MA contributed to data analysis and interpretation and writing of the manuscript.

\section{Ethics approval and consent to participate}

All studies were performed in accordance with the guidelines established by the Tokushima University Committee on Animal Care and Use. The present study was conducted according to the ARRIVE guidelines and AVMA euthanasia guidelines 2013. All experimental protocols were reviewed and approved by the Animal Research Committee of the University of Tokushima.

\section{Patient consent for publication}

Not applicable.

\section{Author information}

Mariko Aoyama, E-mail: aoyama.mariko@tokushima-u.ac.jp

\section{Competing interests}

The authors declare that there are no competing interests that may be perceived as prejudicing the impartiality of the reported research.

\section{References}

1. Kebebew E, Dreenspan FS, Clark OH, Woeber KA and McMillan A: Anaplastic thyroid carcinoma. Treatment outcome and prognostic factors. Cancer 103: 1330-1335, 2005.

2. Tennvall J, Lundell G, Wahlberg P, Bergenfelz A, Grimelius L, Akerman M, Hjelm Skog AL and Wallin G: Anaplastic thyroid carcinoma: Three protocols combining doxorubicin, hyperfractionated radiotherapy and surgery. Br J Cancer 12: 1848-1853, 2002.

3. Sugitani I, Onoda N, Ito K and Sugitabi S: Management of anaplastic thyroid carcinoma: Fruits from ATC Research Consortium of Japan. J Nippon Med Sch 85: 18-27, 2018. 
4. Shimaoka K, Schoenfeld DA, DeWys WD, Creech RH and DeConti R: A randomized trial of doxorubicin versus doxorubicin plus cisplatin in patients with advancer thyroid carcinoma. Cancer 56: 2155-2160, 1985.

5. Ahuja S and Emst H: Chemotherapy of thyroid carcinoma J Endocrinol Invest 3: 303-310, 1987.

6. Ain KB, Egorin MJ and DeSimone PA: Treatment of anaplastic thyroid carcinoma with paclitaxel: Phase 2 trial using ninety-six-hour infusion. Collaborative Thyroid Cancer Health Intervention Trials (CATCHIT) Group. Thyroid 10: 587-594, 2000.

7. Onoda N, Sugino K, Higashiyama T, Kammori, Toda K, Ito K, Yoshida A, Suganuma N, Nakashima N, Suzuki S, et al: The safety and efficacy of weekly paclitaxel administration for anaplastic thyroid cancer patients: A nationwide prospective study. Thyroid 26: 1293-1299, 2016

8. Tahara M, Kiyota N, Yamazaki T, Chayara N, Nakano K, Inagaki L, Toda $\mathrm{K}$, Enokida $\mathrm{T}$, Minami $\mathrm{H}$, Imamura $\mathrm{Y}$, et al: Lenvatinib for anaplastic thyroid cancer. Front Oncol 7: 25, 2017.

9. Hundahl SA, Fleming ID, Fremgen AM and Menck HR: A national cancer data base report on 53,856 cases of thyroid carcinoma treated in the U.S., 1985-1995. Cancer 83: 2638-2648, 1998.

10. Kitamura Y, Shimizu K, Hagahama M, Sugino K, Osaki O, Mimura T, Ito K, Ito K and Tanaka S: Immediate causes of death in thyroid carcinoma: Clinicopathological analysis of 161 fetal cases. J Clin Endocrinol Metab 84: 4034-4039, 1999.

11. Kim S, Park YW, Schiff BA, Doan DD, Yazici Y, Jasser SA Younes M, Mandal M, Bekele BN and Myers JN: An orthotopic model of anaplastic thyroid carcinoma in athymic nude mice. Clin Cancer Res 11: 1713-1721, 2005.

12. Nucera C, Nehs MA, Mekel M, Zhang X, Hodin R, Lawler J, Nose V and Parangi S: A novel orthotopic mouse model of human anaplastic thyroid carcinoma. Thyroid 19: 1077-1084, 2009.

13. Sewell W, Reeb A and Lin RY: An orthotopic mouse model of anaplastic thyroid carcinoma. J Vis Exp 74: 50097, 2013.

14. Paget S: The distribution of secondary growths in cancer of the breast. Lancet 133: 571-573, 1889.

15. Bibby MC: Orthotopic models of cancer for preclinical drug evaluation: Advantages and disadvantages. Eur J Cancer 40: 852-857, 2004.

16. Nehas MA, Nucera C, Nagarkatti SS, Sadow, Morales-Garcia D, Hodin RA and Parangi S: Late intervention with anti-BRAFV600E therapy induces tumor regression in an orthotopic mouse model of anaplastic thyroid cancer. Endocrinology 153: 985-994, 2012.

17. Cha W, Kim DW, Kim SD, Jeon EH, Jeong WJ and Ahn SH Effect of perioperative treatment with a hypoxia-inducible factor-1-alpha inhibitor in an orthotopic surgical mouse models of thyroid cancer. Anticancer Res 35: 2049-2054, 2015.

18. Nehs MA, Nagarkatti S, Nucera C, Hodin RA and Parangi S: Thyroidectomy with neoadjuvant PLX4720 extends survival and decreases tumor burden in an orthotopic mouse models of anaplastic thyroid cancer. Surgery 148: 1154-1162, 2010.
19. Mokhtar M, Kondo K, Takizawa H, Ohtani T, Otsuka H, Kubo H, Kajiura K, Nakagawa Y, Kawanaka Y, Yoshida M, et al: Non-invasive monitoring of anticancer effects of cisplatin on lung cancer in an orthotopic SICD mouse model using [18F] FDG PET-CT. Oncol Rep 31: 2007-2014, 2014.

20. Otani T, Kondo K, Takizawa H, Kajiura K, Fujino H, Otsuka H and Miyoshi H: Non-invasive monitoring of cisplatin and erlotinib efficacy against lung cancer in orthotopic SCID mouse models by small animal FDG-PET/CT and CT. Oncol Rep 41: 447-454, 2019.

21. Marcus C, Whitworth PW, Surasi DS, Pai SI and Subramaniam RM: PET/CT in the Management of thyroid cancers. AJR Am J Roentgenol 202: 1316-1329, 2014.

22. Poisson T, Deandreis D, Leboulleux S, Bidault F, Bonniaud G, Baillot S, Auperin A, AI Ghuzlan A, Travagli JP and Lumbroso J: $18 \mathrm{~F}$-fluorodeoxyglucose positron emission tomography and computed tomography in anaplastic thyroid cancer. Eur J Nucl Med Mol Imaging 37: 2277-2285, 2010.

23. Killkenny C, Browne WJ, Cuthill IC, Emerson M and Altman DG: Improving bioscience research reporting: The ARRIVE guidelines for reporting animal research. Osteoarthritis Cartilage 20: 256-260, 2012.

24. Jing C, Gao Z, Wang R, Yang Z, Shi B and Hou P: Lenvatinib enhances the antitumor effects of paclitaxel in anaplastic thyroid cancer. Am J Cancer Res 4: 903-912, 2017.

25. Ahmed S, Ghazarian MP,Cabanillas ME,Zafereo ME,Williams MD, $\mathrm{Vu}$ T, Schomer DF and Debnam JM: Imaging of anaplastic thyroid carcinoma. AJNR AM J Neuroradiol 3: 547-551, 2018.

26. Deeken-Draisey A, Yang GY, Gao J and Alexiev BA: Anaplastic thyroid carcinoma: An epidemiologic, histologic, immunohistochemical, and molecular single-institution study. Hum Pathol 82: 140-148, 2018.

27. Kostakoglu L and Chauvie S: Metabolic tumor volume metrics in lymphoma. Semin Nucl Med 48: 50-66, 2018.

28. Dibble EH, Alvarez AC, Truong MT, Mercier G, Cook EF and Subramaniam RM: 18F-FDG metabolic tumor volume and total glycolytic activity of oral cavity and oropharyngeal squamous cell cancer: Adding value to clinical staging. J Nucl Med 53: 709-715, 2012.

29. Manohar PM, Beesley LJ, Bellile EL, Worden FP and Avram AM: Prognostic value of FDG-PET/CT metabolic parameters in metastatic radioiodine-refractory differentiated thyroid cancer. Clin Nucl Med 43: 641-647, 2018.

30. Tohyama O, Matsui J, Kodama K, Hata-Sugi N, Kimura T, Okamoto K, Minoshima Y, Iwata M and Funahashi Y: Antitumor activity of lenvatinib (e7080): An angiogenesis inhibitor that targets multiple receptor tyrosine kinases in preclinical human thyroid cancer models. J Thyroid Res 2014: 13, 2014.

31. Miller K, Wang M, Gralow J, Dickler M, Cobleigh M, Perez EA, Shenkiier T, Cella D and Davidson NA: Paclitaxel plus bevacizumab versus paclitaxel alone for metastatic breast cancer. N Engl J Med 26: 2666-2676, 2007. 